\title{
Improvement of Output Impedance Modulation Effect of High Speed DAC
}

\author{
Dongmei Zhu a, Xiaodan Zhou ${ }^{\text {b }}$, Jun Liu c, Luncai Liu, Dayong Pu \\ Sichuan Institute of Solid State Circuits China Electronics Technology Group Corp. \\ Chongqing, China. \\ a13896000372@163.com, bdavidaayaa@126.com, cdliujun_421@126.com
}

\begin{abstract}
High speed digital to analog converter (DAC) are always used in signal processing system to accomplish the signal reconstruction, where the spurious free dynamic range (SFDR) of the system is especially important. The SFDR determines the ability of DAC and the system to distinguish the carrier signal from other spurs. By increasing the output impedance of the high speed DAC will help to lower the nonlinearity, thus improve the SFDR. This paper describes the technique which will reduce the nonlinearity by increasing the output impedance of the DAC, which can achieve 14 bit resolution and 1.2GSPS, and SFDR $\geq 76 \mathrm{dBc} @ \mathrm{FDAC}=1.2 \mathrm{GHz}, \mathrm{FOUT}=50 \mathrm{MHz}$.
\end{abstract}

Keywords: DAC; SFDR; output impedance; nonlinearity distortion.

\section{Introduction}

With the rapid development of microelectronics, and the aid of advanced processes such as Nano-CMOS, new generation SiGe and silicon based compound Heterogeneous, modern RF DACs generally adopt $0.18 \sim 0.13 \mu \mathrm{m}$ CMOS or SiGe BiCMOS process. Since 2014, the 12 16 bits DACs whose conversion rate beyond $1 \mathrm{Gsps}$ have adopted $65 \mathrm{~nm}$ CMOS process. It is expected that in the next 3 years there will be DACs which adopt 40nm or 28nm CMOS process. Compared with conventional high speed DACs whose output can only be within baseband Nyquist zone, there are more and more modern Gsps RF DACs which have mixed mode and can support output within high Nyquist zone. In the future, these RF DACs together with RF ADCs can implement direct radio signal sampling and synthesis which is required by Software Radio. With the widely application of JESD204B high speed serial interface, the performance and speed of high speed DACs with high density and multichannel will continue to improve.

High speed broadband DAC are often used in broadband communication system, automatic test device, radar and instrument to reconstruct the signal, where the frequency domain performance of these signal processing systems are of most important. Therefore, the focus of these systems is on the frequency domain parameter SFDR. The SFDR is determined by the nonlinearity of the DAC, and reduction of it is the key technique in a high speed DAC design.

\section{Circuit Implementation}

\subsection{Design Consideration}

Segmented current steering architecture is often used in high speed ADC, as can be seen in Fig1. It always includes high speed data interface, decoder, switch driver, current source array, SPI, digital calibration, reference, amplifier buffer and so on. 


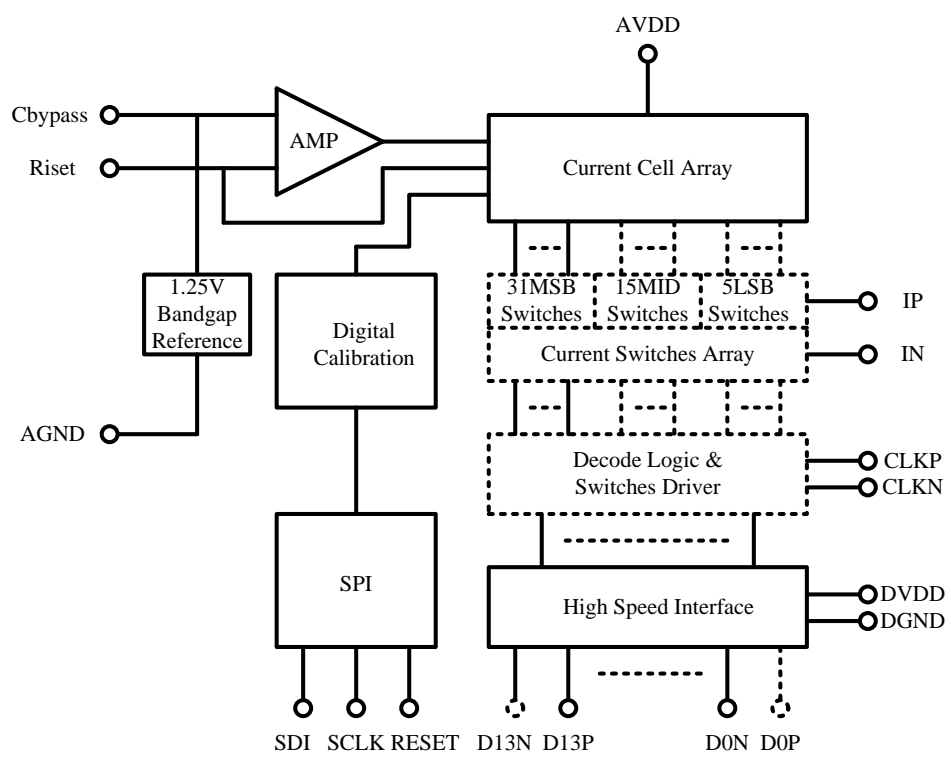

Fig. 1 Architure of 14 bit Current Steering DAC

\subsection{Design Challenges}

The most important parts are current source array and current switch design. The output stage of high speed current steering DACs usually adopt the circuit structure which can be seen in Figure 2.

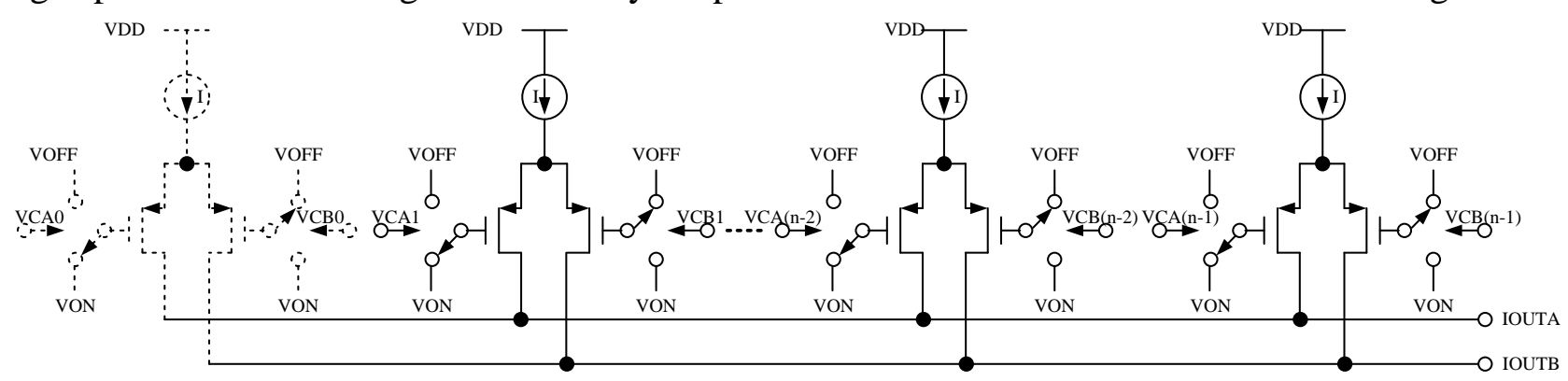

Fig. 2 High Speed Current Steering DAC Output Stage

The challenges are mismatch of current source array cell, and differential pairs of current switch, finite output impedance of current source and switch, output voltage waveform distortion caused by nonlinear capacitance of output, interference or modulation of sensitive bias voltage, the source capacitance of differential current switch, coupling of switch, and so on. The dynamic large signal change caused by high and low level, slope and crossing of the driving signal on the gate of current switch differential pairs, will introduce static and dynamic nonlinearity. The mismatch of switching time between the current steering cell caused by random and systematic mismatch, and the voltage change of source node of the current switch differential pairs caused by the feedback of output signal, both will introduce dynamic nonlinearity, as can be seen in Fig.3.

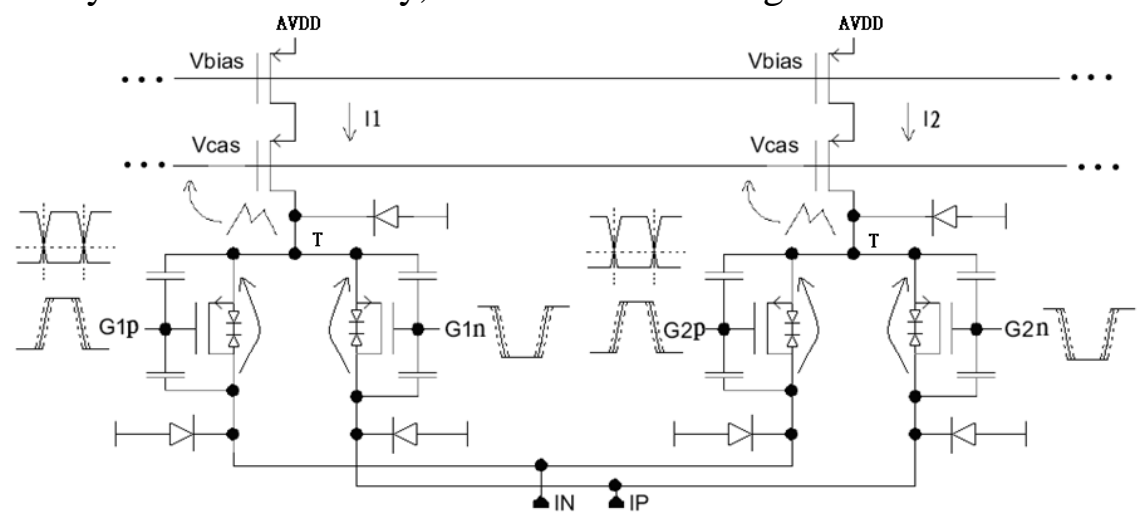

Fig. 3 Nonideal Source of Current Source and Current Switch of High Speed DAC 
Assuming that the output resistance of current source is ro, when inputting digital code $\mathrm{D}$, there will be x current sources shunting at output. Next, provided the external resistance at the output of $\mathrm{DAC}$ is RT, then the output resistance shown in Figure 2 is equivalent to Figure 4.

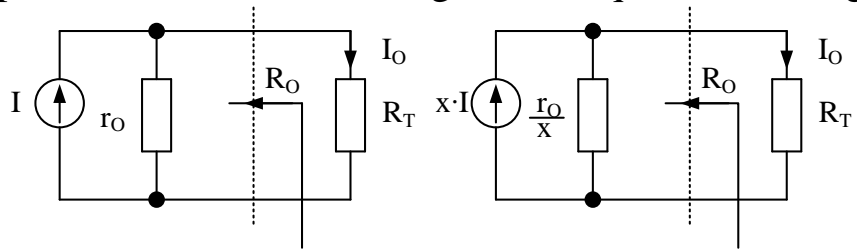

Fig. 4 Output Resistance Equvialent Circuit of Current Source

As can be seen in Figure 4, the input digital code will modulate the output resistance of DAC. The effect of output resistance modulated by input code will introduce nonlinearity distortion to the DAC, and then affect key parameters like integral nonlinearity (INL) error and SFDR. Therefore, for the high speed and ultra-high speed DAC design, how to reduce the effect of output resistance modulated by input code is one of important means to improve SFDR.

\subsection{Circuit Architecture}

To increase the output impedance of current source is one of the most important approach to reduce nonlinearity, and is implemented effectively by cascode current mirror and active cascode Conventional cascode will occupy more headroom which can reduce the swing severely. Instead, the more effective active cascode can be utilized as shown in Fig.5. And this way can also suppress the noise on bias voltage coupling from switch spur in conventional cascode.
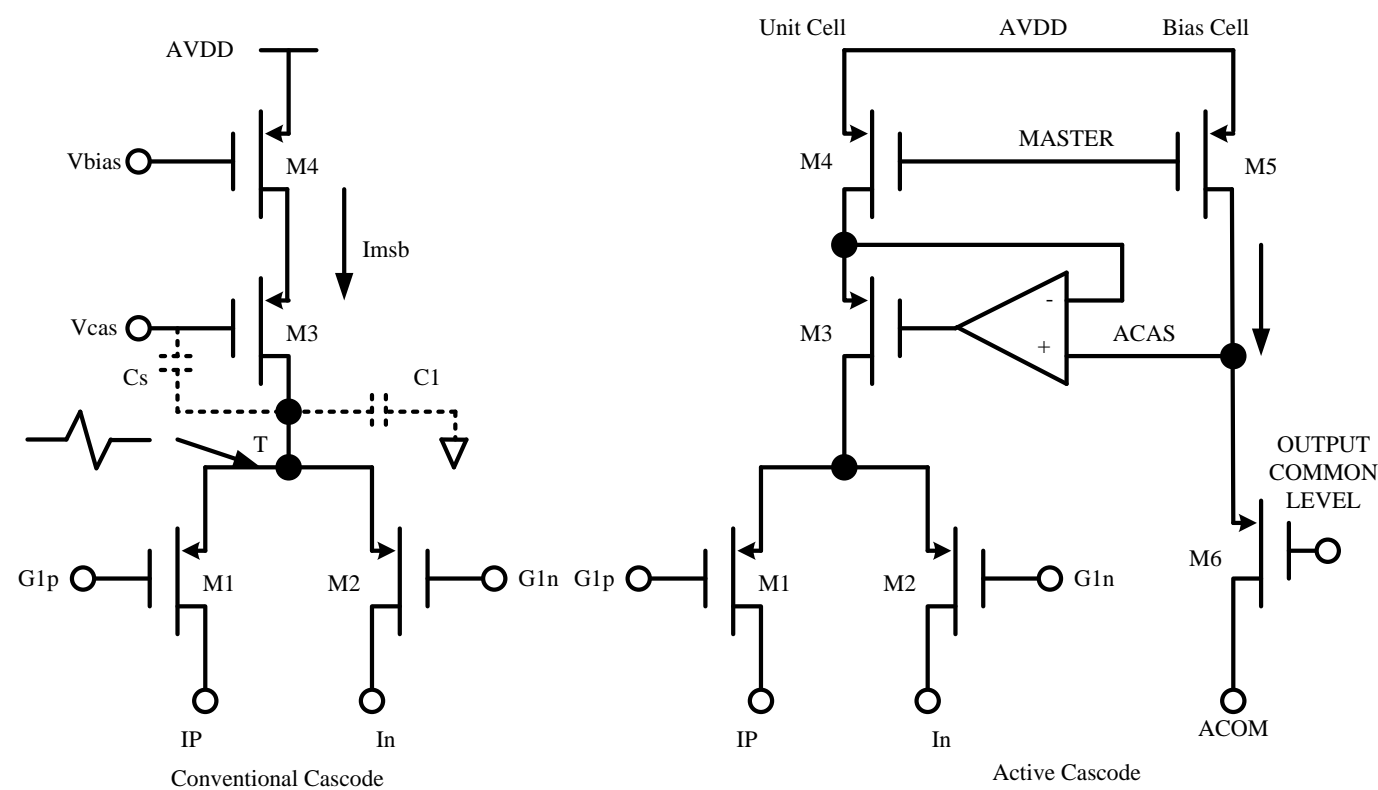

Fig. 5 Active Cascode Circuit

The architecture of conventional current steering output stage can be seen in Fig.6. VON is the low level causing M1, M2 to conduct, and VOFF is the high level to turn off M1, M2. VON and VOFF should be at the allowable or desire level on output node IP and IN. 


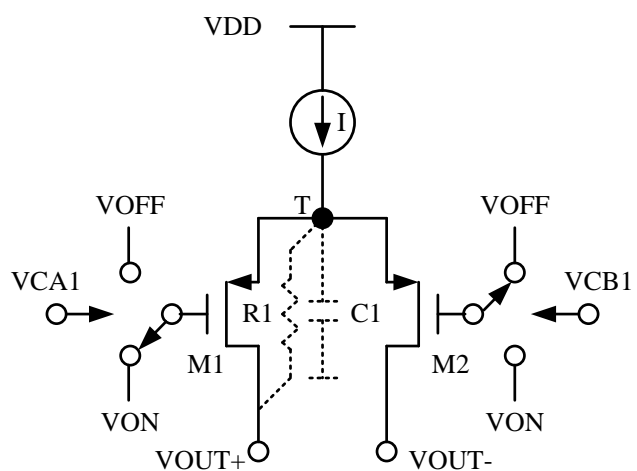

(a)

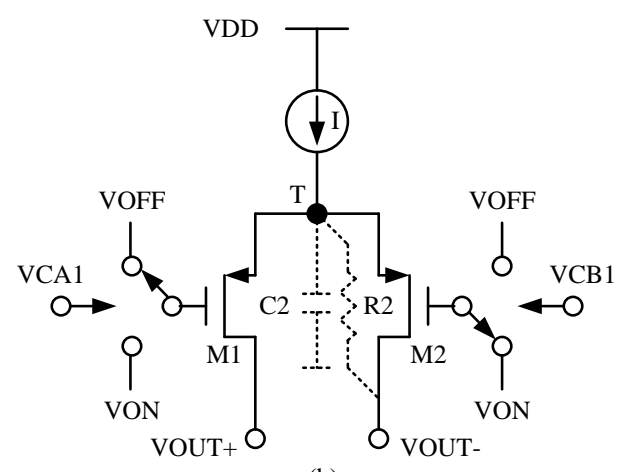

(b)

Fig. 6 Steering of Output Stage

In Fig.6, when output is in state (a), M1 is on, M2 is turned off. When M1 is remaining on, there is a parasitic resistance $\mathrm{R} 1$, and because there is a parasitic junction capacitance of MOS transistor, output node Vout+ will charge C1 through R1 when M1 is on. Conversely, when output is in state (b), M2 is on, and output node Vout- will charge C2 through R2. When output is switching between state (a) and (b), the potential of node $\mathrm{T}$ will change corresponding, thus the output impedance of current source I will change corresponding.

It can use output feedback to reduce the influence of code modulation of output impedance, as can be seen in Fig.7.

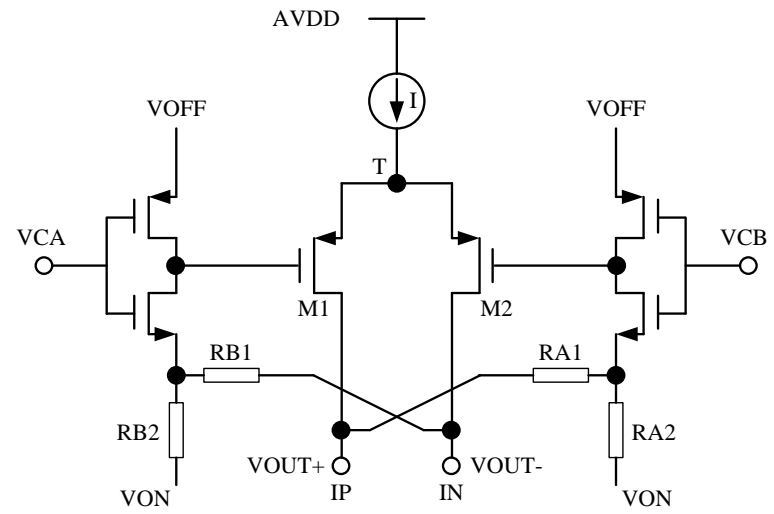

Fig. 7 Steering of Output Stage

In Fig.7, it can greatly adjust the current switch M1 and M2 by feed backing differential signal on output node IP and IN. By this way, the signal change on output node IP and IN will not inference the voltage on current source common node $\mathrm{T}$. It means that there is no charge transfer and distortion relevant to signal on output node IP and IN, thus improve the SFDR of DAC.

\subsection{Circuit Technique}

Assuming that the current switch cell M1 is on by the control signal VCA, and M2 is turned off by VCB. If there is voltage rise on output node IP, then the voltage of common node $T$ will rise to follow output node IP because of the finite output impedance of M1 and the parasitic capacitance of common node T. Meanwhile, because of voltages on IP and IN are differential, the voltage on output node IN will fall. As can be seen in Fig.7, by a appropriate scaling feedback circuit, the falling voltage of node IN will be connected to the gate of M1, which cause the gate voltage of M1 to drop, then the voltage of common node $\mathrm{T}$ will drop as the source voltage is always following the gate voltage of a MOS transistor, and this eliminates the interference of voltage rise of output node IP to common node T. The scale factor of the feedback circuit is design to prevent the voltage of common node $\mathrm{T}$ from following the output node IP. The result is that the current source has adequate and improved output impedance when M1 is on.

As before, when M2 is turned on by control signal VCB, VCA will turned off M1, and if the voltage on common node $\mathrm{T}$ is rising by the voltage rise on output node $\mathrm{IN}$, the feedback circuit will 
feedback the falling voltage of output node IP to make the gate voltage of M2 drop, which will cause the voltage of common node $\mathrm{T}$ to drop by the source following effect of M2.

By the scaling feedback circuit of the control cell of M1 and M2, the voltage of output node will no longer be disturbed by output node IP and IN, as if the circuit has infinite output impedance.

\subsection{Circuit Principle}

Assume that the voltage change of output node IP is $\mathrm{V}_{\mathrm{IP}}$, the trans conductance of $\mathrm{M} 1$ is $\mathrm{g}_{\mathrm{m}-\mathrm{M} 1}$, the drain-source trans conductance is $g_{d s-M 1}$, the output impedance is $r_{o}$, and $r_{o}=1 / g_{d s-M 1}$, the change of the M1 source voltage is $\mathrm{v}_{\mathrm{T} 1}$, when there is no feedback circuit, then

$v_{I P}=v_{T 1} \cdot g_{m-M 1} \cdot r_{o}$

Which can get that

$v_{T 1}=\frac{v_{I P} \cdot g_{d s-M 1}}{g_{m-M 1}}$

It is the relationship between common node voltage and output node voltage.

When there is a feedback circuit, as the voltage change of output node IN is opposite to output node IP, then $v_{I N}=-v_{I P}$, and the gate voltage of M1 is

$$
\begin{aligned}
V_{G-M 1} & =V_{O N}+\left(v_{I N}-V_{O N}\right) \cdot K_{b} \\
& =V_{O N}+v_{I N} \cdot K_{b}-V_{O N} \cdot K_{b} \\
& =V_{O N}-v_{I P} \cdot K_{b}-V_{O N} \cdot K_{b} \\
& =V_{O N} \cdot\left(1-K_{b}\right)-v_{I P} \cdot K_{b}
\end{aligned}
$$

Where is $\mathrm{K}_{\mathrm{b}}$ is

$$
K_{b}=\frac{R B 2}{R B 1+R B 2}
$$

According to the source following principle, $\mathrm{v}_{\mathrm{T} 2}$ is

$v_{T 2}=-v_{I P} \cdot K_{b}$

To make voltage of common node $\mathrm{T}$ unchanged, then

$v_{T 1}+v_{T 2}=0$

It means that

$$
V_{I P} \cdot K_{b}=\frac{V_{I P} \cdot g_{d s-M 1}}{g_{m-M 1}}
$$

Therefore, the $K_{b}$ should be

$$
K_{b}=\frac{R B 2}{R B 1+R B 2}=\frac{g_{d s-M 1}}{g_{m-M 1}}
$$

\section{Chip Implement}

This 14 bit $1.2 \mathrm{GSPS}$ DAC improves the linearity by reduce the code modulation effect on the output impedance. The DAC is fabricated in $0.18 \mu \mathrm{m} 1 \mathrm{P} 6 \mathrm{M}$ CMOS process, and the area is $5.9 \mathrm{~mm} \times 6.1 \mathrm{~mm}$, the die photo can be seen in Fig.9. 


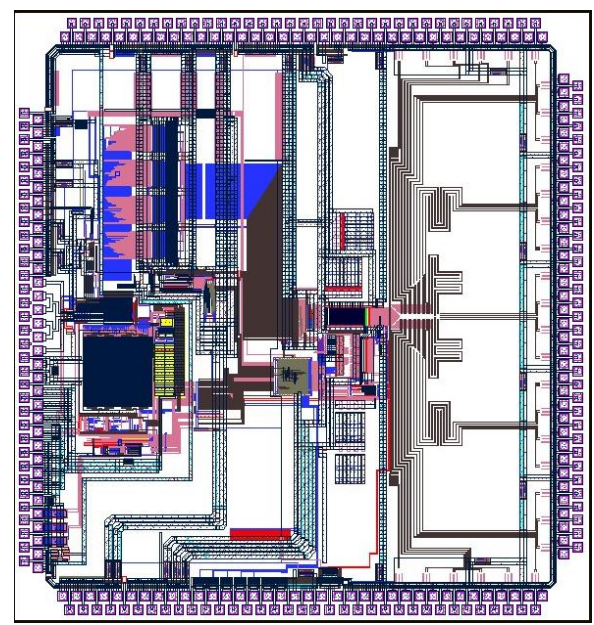

Fig. 8 Die photo of a 14 bit 1.2GSPS DAC

\section{Measured Result}

The DAC is measured under $1200 \mathrm{MHz}$ clock, $50 \mathrm{MHz}$ and $550 \mathrm{MHz}$ input, the measure result can be seen in Fig.10 and Fig.11.

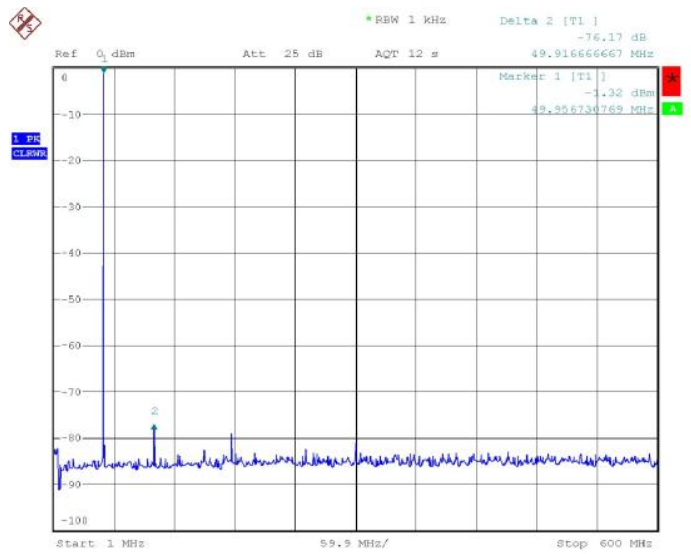

Fig. 9 Measured result @ $\mathrm{f}_{\mathrm{IN}}=50 \mathrm{MHz}, \mathrm{SFDR}=76 \mathrm{dBc}$

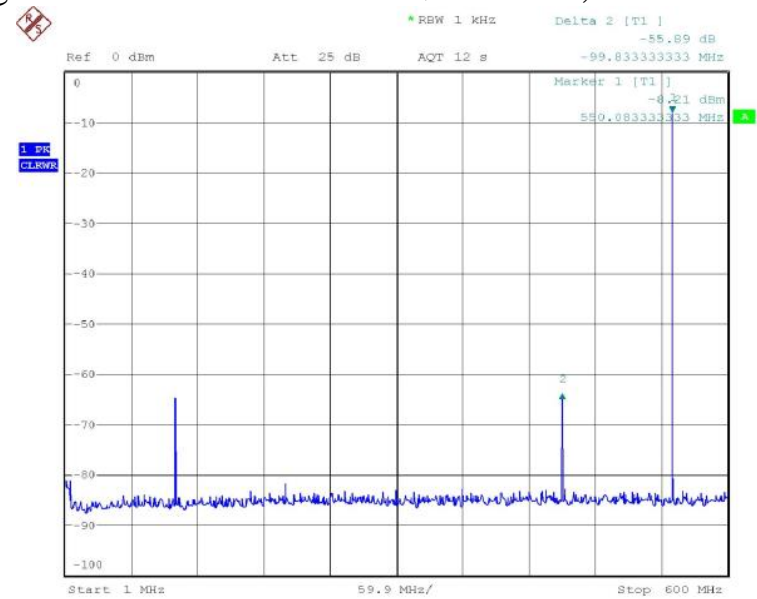

Fig. 10 Measured result @ $\mathrm{f}_{\mathrm{IN}}=550 \mathrm{MHz}, \mathrm{SFDR}=55 \mathrm{dBc}$

\section{Summary}

In high speed DAC design, improving the SFDR by reducing the code modulation effect on the output impedance is a very effective method. This paper implements a 14bit 1.2GSPS DAC by this technique, which achieves SFDR $\geq 76 \mathrm{dBc} @ \mathrm{~F}_{\mathrm{DAC}}=1.2 \mathrm{GHz}, \mathrm{F}_{\text {OUT }}=50 \mathrm{MHz}$. 


\section{References}

[1] C.-H. Lin et al, “A 12-bit 2.9GS/s DAC with IM3<-60dBc beyond $1 \mathrm{GHz}$ in 65nm CMOS”, IEEE JSSC, Vol.44, Dec.2009, pp.3285-3293.

[2] S. Spiridon et al., "A $375 \mathrm{~mW}$ Multimode DAC-Based Transmitter with $2.2 \mathrm{GHz}$ Signal Bandwidth and In-Band IM3 < -58dBc in 40nm CMOS", IEEE JSSC, Vol.48, Jul. 2013, pp.1595-1604.

[3] Douglas A. Mercer, "Low-Power Approaches To High - Speed Current - Steering Digital - to -

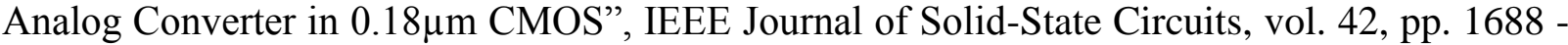
1698, August 2007.

[4] Bernd Schafferer, Richard Adams, "A 3V CMOS 400mW 14b 1.4GS/s DAC for multi-carrier applications", IEEE International Solid-State Circuits Conference, vol. XLVII, pp. 360 - 361, February 2004.

[5] Zhu Dongmei, "A digital static calibration technology for 16-bit current steering DAC", Sichuan Institute of Solid-state Circuit, 2009.

[6] P. Andricciola and H. P. Tuinbut, "The temperature dependence of mismatch in deep submicrometer bulk MOSFETs,” IEEE Electron Device Lett, vol.30, no.6.pp.690-692, 2009. 